\title{
Caracterização de Cuidadores de Crianças e Adolescentes Estomizados Atendidos em Serviço de Reabilitação
}

\section{Characterization of Caregivers of Ostomized Children and Teenagers Treated in Service Rehabilitation}

\section{Caracterización de los Cuidadores de Niños y Adolescentes Estomizados Atendidos en el Servicio de Rehabilitación}

\author{
Sandra de Nazaré Costa Monteiro' ${ }^{1}$ Manuela Costa Melo', Ivone Kamada ${ }^{1}$, Ana Lúcia da Silva
}

\begin{abstract}
RESUMO
Introdução: O cuidador é definido como aquele que presta cuidados a pessoas que não podem fazê-lo sozinhas. Neste estudo, o cuidador assiste indivíduos com estomias intestinais e/ou urinárias. Objetivo: Identificar as características sociodemográficas e clínicas de cuidadores de crianças e adolescentes estomizados atendidos em um programa de reabilitação da rede pública do Distrito Federal. Métodos: Trata-se de um estudo transversal, descritivo e com abordagem quantitativa, cuja coleta de dados foi realizada no período de abril a junho de 2013. A amostra foi constituída de 22 cuidadores de crianças e adolescentes estomizados. Foi realizada análise estatística com distribuição de frequência e percentual e os resultados articulados com o referencial teórico. Resultados: Identificou-se que a média de idade dos cuidadores foi de 41,27 anos, predomínio do gênero feminino, casados, que estudaram até o ensino fundamental e estavam desempregados. A maioria referiu possuir algum problema de saúde. Relataram receber benefício do governo e ter custos adicionais com fraldas, medicamentos e dispositivos coletores. O tempo na função de cuidador variou de 15 dias a 14 anos e três meses. Conclusão: Constatou-se neste estudo que os cuidadores podem apresentar problemas na saúde física e emocional em decorrência de priorizarem o cuidado da criança e/ou adolescente estomizado.
\end{abstract}

DESCRITORES: Estomaterapia. Cuidadores. Crianças. Estomas Cirúrgicos.

\begin{abstract}
Introduction: The caregiver is defined as one that provides care to people who cannot do it, in this study the caregiver assists individuals with intestinal stomas and / or urinary. Objective: Identify the sociodemographic characteristics and clinics of caregivers of ostomized children and adolescentsattending a rehabilitation program, from the public network of the Federal District. Methods: This is a transversal, descriptive study, with a quantitative approach, in which the data collection was carried out from April to June 2013. The sample consisted of 22 caregivers of ostomized children and adolescents. Statistical analysis with frequency and percentage distribution was performed, and the results were articulated to the theoretical framework. Results: It was found that the average age of caregivers was 41.27 years, predominantly female, married, who studied up to elementary school and were unemployed. The majority reported having some health problem. They also reported they receive money from the government and have additional costs for diapers, medicines and collector devices. The time on the caregiver function ranged from 15 days to 14 years and three months. Conclusion: It was found in the study that caregivers can present problems in the physical and emotional health, as a result of prioritizing child care and / or ostomy teenager
\end{abstract}

DECRIPTORS: Stomatherapy. Caregivers. Child. Surgical Stomata.

Programa de Pós Graduação em Ciências da Saúde, Universidade de Brasília (UnB) - Brasília (DF), Brasil.

Endereço para correspondência: SCRN 708/09 Bloco D, apto. 303B - Asa Norte - CEP: 70741-640 - Brasília (DF), Brasil. E-mail: melomanuela91@gmail.com Artigo recebido em: 12/04/2015 - Aceito para publicação em: 23/08/2015 


\section{RESUMEN}

Introducción: El cuidador se define como aquel que presta atención a las personas que no pueden hacerlo solas. En este estudio el cuidador asiste a individuos con ostomías intestinales y/o urinarias. Objetivo: Identificar las características sociodemográficas y clínicas de los cuidadores de niños y adolescentes ostomizados atendidos en un programa de rehabilitación, de la red pública de Distrito Federal. Métodos: Se trata de un estudio transversal, descriptivo y con enfoque cuantitativo, cuyos datos fueron recogidos en el período de abril a junio de 2013. La muestra fue compuesta por 22 cuidadores de niños y adolescentes ostomizados. Fue realizado análisis estadístico con distribución de frecuencia y porcentual, y los resultados articulados con la referencia teórica. Resultados: Fue identificado que la edad promedio de los cuidadores era de 41,27 años, predominio del género femenino, casados, que estudiaron hasta la escuela primaria y estaban desempleados. La mayoría mencionó tener algún problema de salud. Reportaron haber recibido beneficio del gobierno y tener costos adicionales con pañales, medicamentos y dispositivos colectores. El tiempo en el papel de cuidador osciló entre 15 días a 14 años y tres meses. Conclusión: El estudio constató que los cuidadores pueden presentar problemas en la salud física y emocional como resultado de priorizar el cuidado del niño y/o adolescente ostomizado.

DESCRIPTORES: Estomaterapia. Cuidadores. Niño. Estomas Quirúrgicas

\section{INTRODUÇÃO}

A estomia é uma situação que atinge pessoas de todas as idades. O procedimento para a confecção da mesma é potencialmente acompanhado de complicações que podem ser evitadas com o planejamento do local de sua confecção e com o uso de técnica cirúrgica adequada, entre outros cuidados. As estomias são confeccionadas para utilização de forma temporária ou permanente e, nesse período, requer adaptação ${ }^{1,2}$. Para a adaptação, faz-se necessário que o enfermeiro especializado nos cuidados com estomia encoraje a família a encontrar seu modo de participar dos cuidados diários e oferecer ao estomizado a oportunidade de desenvolver suas possibilidades diante das modificações que essa condição proporciona na vida ${ }^{1,3}$.

Crianças e adolescentes podem adquirir um estoma por diversas causas, as mais frequentes são as anomalias congênitas, podendo ser necessária por diferentes razões, como desvio, descompressão ou acesso ao lúmen intestinal, como no caso de gastrostomias e jejunostomias. Normalmente, as estomias são confeccionadas quando há perfuração, isquemia e/ou a derivação for considerada insegura, a ileostomia proximal é utilizada para proteger a anastomose distal após proctocolectomia restauradora para polipose familiar ou colite ulcerosa. Da mesma forma, é utilizada a colostomia, tanto antes quanto depois de um procedimento de resgate para megacólon congênito ou ânus imperfurado ${ }^{2,4,5}$. As derivações urinárias também congregam a confecção de estomas, denominados urostomias e são oriundas de causas obstrutivas da pelve renal, congênitas incluindo as malformações da coluna lombossacral, válvula de uretra posterior e mielomeningocele, além das adquiridas por trauma ou manipulação excessiva em cirurgias ${ }^{6}$.

Os cuidadores de crianças e adolescentes vivenciam o enfrentamento de seus filhos à condição de serem ou estarem estomizados. De certa forma, a família e o cuidador, diante desse processo de adaptação, precisam repensar a sua estrutura e forma organizacional enquanto grupo, porque o cuidado à criança e/ou adolescente estomizado exige a presença constante de um cuidador ${ }^{8,10}$. Esse cuidador muitas vezes não tem a opção de decidir acerca do papel que assumirá, pois o sistema familiar, a partir de características geracionais, legados, mitos e regras já o condicionam como o cuidador principal ${ }^{9}$. Apesar de desempenharem um papel importantíssimo para minimizar o sofrimento e auxiliar o bem-estar, os cuidadores são marginalizados, seu trabalho é pouco valorizado e, além disso, não são reconhecidos como pessoas que estão passando por um processo doloroso e que precisam de ajuda, apoio e orientação ${ }^{11-12}$.

A justificativa deste estudo está na possibilidade de fornecer subsídios aos profissionais e gestores de saúde para que possam planejar, sistemática e individualmente, as atividades do programa de reabilitação e, assim, oferecer assistência qualificada, além de criar a possibilidade de fomento para estudos futuros nessa temática. Diante do exposto, este 
estudo objetivou identificar as características sociodemográficas e clínicas de cuidadores de crianças e adolescentes estomizados atendidos em um programa de reabilitação do Distrito Federal.

\section{MÉTODOS}

Trata-se de estudo descritivo, abordagem quantitativa, desenvolvido em um programa de reabilitação no período de abril a junho de 2013. O programa é desenvolvido em um hospital de cuidados terciários, localizado na cidade de Brasília (DF), que atende as diretrizes do Sistema Único de Saúde (SUS).

No DF, o cuidado ao estomizado oferecido na rede pública é gerenciado pelo Núcleo de Atenção Básica do Estomizado, da Secretaria de Estado de Saúde (SES), sendo que no ano de 2012, havia 785 pessoas estomizadas cadastradas, dentre crianças, adolescentes e adultos ${ }^{7}$.

A população do estudo foi composta por conveniência, desde que os sujeitos estivessem atuando como cuidadores de crianças e adolescentes estomizados com idade entre $8 \mathrm{e}$ 18 anos no período da coleta de dados e que já estivessem há mais de seis meses prestando os cuidados. Outros critérios de inclusão previamente definidos foram os seguintes: pais e/ou responsáveis que ressaltaram ser os principais cuidadores da criança e/ou adolescente no lar e na instituição, sem limitação cognitiva e que aceitaram participar da pesquisa.

Os cuidadores selecionados foram recrutados de três maneiras. Primeiro, por meio do cadastro do Núcleo de Atenção ao Estomizado, em que havia 37 crianças cadastradas no sistema, porém somente oito cuidadores de estomizados atenderam os critérios de seleção. Segundo, pelo sistema de prontuário eletrônico Track care ${ }^{\circledast}$, em que foram localizados mais 11 indivíduos, sendo que todos aceitaram participar do estudo. Terceiro, no cadastro do atendimento aos estomizados no Hospital Materno Infantil de Brasília (HMIB), dos 27 estomizados, apenas três aceitaram participar. No total, a amostragem foi composta por 22 cuidadores de crianças e adolescentes estomizados.

Todos os participantes foram convidados individualmente, e, após concordarem em participar, foram esclarecidos quanto aos objetivos e procedimentos do estudo por meio da leitura do Termo de Consentimento Livre e Esclarecido (TCLE). Posteriormente, foram retiradas as dúvidas que emergiram e, apenas após esse processo, era solicitada sua anuência mediante assinatura do TCLE. A coleta de dados foi realizada na residência do próprio cuidador e na unidade de cirurgia pediátrica do HMIB.

O instrumento utilizado contemplou questionário estruturado, contendo dados elaborados para a presente pesquisa. Utilizou-se as seguintes variáveis sociodemográficas: idade, gênero, situação conjugal, cor da pele, grau de instrução, ocupação e renda per capita; e as variáveis clínicas: problemas de saúde conhecido, uso de medicamentos, consulta médica no último ano e dados referentes à função de cuidador. $\mathrm{O}$ tempo para aplicação de cada instrumento variou de 20 a 40 minutos.

Os dados foram analisados utilizando-se o programa estatístico Statistical Package for the Social Sciences (SPSS) $20.0^{13}$. Os dados foram compilados por meio da técnica de estatística descritiva e, posteriormente, organizados na forma de tabelas com frequências absolutas e relativas.

O estudo recebeu aprovação do Comitê de Ética em Pesquisa (CEP) da Fundação de Ensino e Pesquisa em Ciências da Saúde da SES/DF, em cumprimento à Resolução no 466/2012, do Ministério da Saúde, que versa sobre pesquisa envolvendo seres humanos sob o Protocolo no 251.308 de 22 de abril de 2013.

\section{RESULTADOS}

A amostra analisada caracterizou-se por 22 cuidadores, houve predominância do sexo feminino 90,9\%, com destaque para a média de idade de 41,27 anos. Com relação à procedência, evidenciou-se que $63,6 \%$ residiam em áreas administrativas do DF, em contrapartida $36,3 \%$ eram provenientes de outros estados da Federação, tais como Goiás, Bahia e Maranhão. As crianças e os adolescentes estomizados encontravam-se na faixa etária de 11 a 15 anos de idade $(63,3 \%)$, sendo que a maioria $(59,09 \%)$ apresentava estomias temporárias. Em relação ao tipo de estomia, 44\% apresentava colostomia; $32 \%$, ileostomia; seguidos de $12 \%$, urostomia; 6\%, gastrostomia; e 3\%, jejunostomia.

Em observância aos dados, conforme a Tabela 1,77,2\% dos cuidadores entrevistados se autodeclararam de cor parda. Com relação à situação conjugal, $50 \%$ estavam casados ou moravam juntos, seguida de $31,8 \%$ que eram separados ou ficaram viúvos, sendo que o tempo de casado ou de que moravam juntos variou de 6 meses a 34 anos, com média de 16 anos. No que se refere ao grau de instrução, 50\% 
possuíam o ensino fundamental, sendo que desses, 40,9\% não haviam concluído. Quanto à ocupação, 45,5\% estavam desempregados, e a renda per capita média variou de 1 a 2 salários mínimos para $81,8 \%$. O número de filhos variou de 1 a 9, com média de 3,6 filhos por cuidador.

De acordo com a situação de saúde de cuidadores, verificou-se que a maioria $(72,7 \%)$ referiram algum problema de saúde, sendo que $45,4 \%$ utilizavam medicamentos para

Tabela 1. Características socioeconômicas e demográficas de cuidadores de crianças e adolescentes estomizados, Brasília (DF), Brasil; $2013(n=22)$.

\begin{tabular}{|c|c|c|}
\hline \multirow{2}{*}{ Variáveis } & \multicolumn{2}{|c|}{ Distribuição } \\
\hline & $\mathrm{n}$ & $\%$ \\
\hline \multicolumn{3}{|l|}{ Cor da pele } \\
\hline Branca & 2 & 9,1 \\
\hline Negra & 3 & 13,6 \\
\hline Parda & 17 & 77,2 \\
\hline \multicolumn{3}{|l|}{ Situação Conjugal } \\
\hline Casado & 11 & 50,0 \\
\hline Separado/viúvo & 7 & 31,8 \\
\hline Solteiro & 4 & 18,2 \\
\hline \multicolumn{3}{|l|}{ Grau de instrução } \\
\hline Fundamental incompleto & 9 & 40,9 \\
\hline Fundamental completo & 2 & 9,1 \\
\hline Médio incompleto & 5 & 22,7 \\
\hline Médio completo & 4 & 18,2 \\
\hline Superior incompleto & 2 & 9,1 \\
\hline \multicolumn{3}{|l|}{ Ocupação } \\
\hline Desempregado & 10 & 45,5 \\
\hline Empregado & 7 & 31,8 \\
\hline Do lar & 4 & 18,2 \\
\hline Autônomo & 1 & 4,5 \\
\hline \multicolumn{3}{|l|}{ Renda per capita familiar* } \\
\hline Até 1 & 9 & 40,9 \\
\hline $2-3$ & 9 & 40,9 \\
\hline $4-5$ & 4 & 18,2 \\
\hline Total & 22 & 100,0 \\
\hline
\end{tabular}

*salários mínimos controle do problema. Considerando a procura por atendimento médico no último ano, neste estudo $36,4 \%$ informou não ter feito essa busca, em contrapartida $27,3 \%$ dos participantes faziam acompanhamento, retornaram ou procuraram por atendimento médico, pelo menos, duas vezes, conforme os dados apresentados na Tabela 2.

A Figura 1 mostra os problemas de saúde que acometeram os cuidadores. Houve predominância de $60 \%$ das pessoas com problemas circulatórios, respiratórios e ortopédicos. Dentre as doenças referidas pelos cuidadores encontrou-se: asma brônquica, bronquite, intolerância à glucose, hipotensão arterial, hipertensão arterial, problemas na próstata, afecções renais, polipose adenomatosa familiar, reumatismo, problemas na coluna vertebral, dor no pé por esporão e linfonodos axilares.

Em relação a receberem ajuda financeira, a Tabela 3 mostra que $81,8 \%$ receberam benefício do governo, como: auxílio para tratamento da doença (49,5\%), aposentadoria $(9,1 \%)$, bolsa família $(13,5 \%)$, benefício de prestação continuada $(4,5 \%)$ e seguro desemprego $(4,5 \%)$. Dos sujeitos entrevistados, $81,8 \%$ relatou ter faltado compromisso pessoal ou profissional para cuidar de seu filho. No que se refere aos custos com o cuidado, 72,7\% afirmou ter custos

Tabela 2. Características clínicas de cuidadores de crianças e adolescentes estomizados, Brasília (DF), Brasil; 2013 ( $n=22)$.

\begin{tabular}{|c|c|c|}
\hline \multirow{2}{*}{ Variáveis } & \multicolumn{2}{|c|}{ Distribuição } \\
\hline & $\mathrm{n}$ & $\%$ \\
\hline \multicolumn{3}{|c|}{ Possui algum problema de saúde } \\
\hline $\operatorname{sim}$ & 16 & 72,7 \\
\hline Não & 6 & 27,3 \\
\hline \multicolumn{3}{|c|}{ Uso de medicamentos } \\
\hline $\operatorname{sim}$ & 10 & 45,4 \\
\hline Não & 12 & 54,5 \\
\hline \multicolumn{3}{|c|}{ Consultou médico no último ano } \\
\hline Não & 8 & 36,4 \\
\hline $1 \mathrm{vez}$ & 3 & 13,6 \\
\hline 2 vezes & 6 & 27,3 \\
\hline 3 vezes & 1 & 4,5 \\
\hline $4 \mathrm{ou}+$ & 4 & 18,2 \\
\hline Total & 22 & 100,0 \\
\hline
\end{tabular}




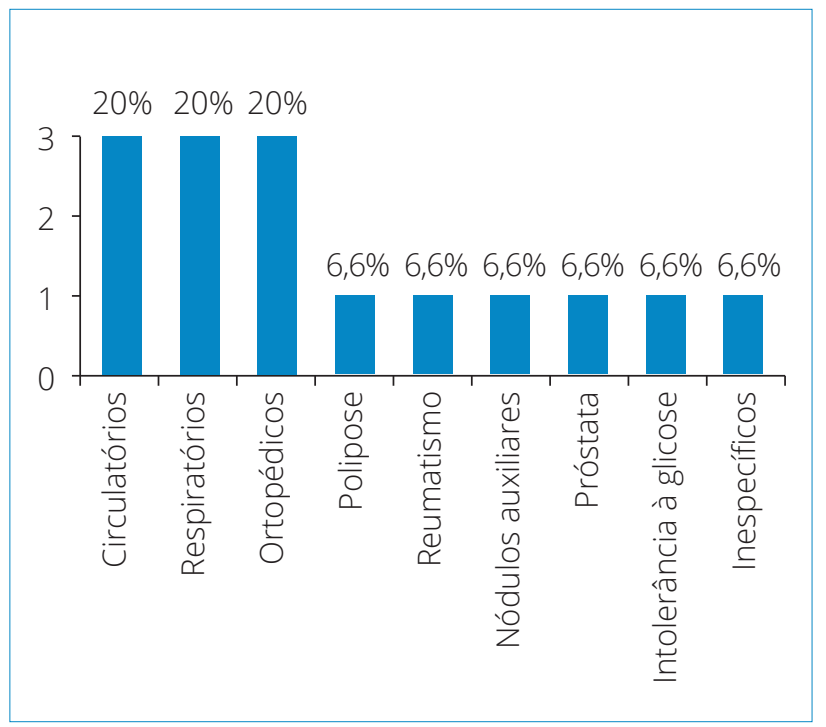

Figura 1. Distribuição de cuidadores de crianças e adolescentes estomizados atendidos em um serviço de reabilitação do Distrito Federal, de acordo com os prolemas de saúde relatados. Brasília (DF) Brasil; 2013.

Tabela 3. Características socioeconômicas e demográficas de cuidadores de crianças eadolescentes estomizados. Brasília (DF), Brasil; 2013 ( $n=22)$.

\begin{tabular}{|c|c|c|}
\hline \multirow{2}{*}{ Variáveis } & \multicolumn{2}{|c|}{ Distribuição } \\
\hline & $\mathrm{n}$ & $\%$ \\
\hline \multicolumn{3}{|c|}{ Ajuda financeira para o cuidado } \\
\hline Sim & 18 & 81,8 \\
\hline Não & 4 & 18,1 \\
\hline \multicolumn{3}{|c|}{ Faltou emprego para cuidar } \\
\hline $\operatorname{sim}$ & 18 & 81,8 \\
\hline Não & 4 & 18,1 \\
\hline \multicolumn{3}{|c|}{ Custos com a função de cuidador } \\
\hline $\operatorname{sim}$ & 16 & 72,7 \\
\hline Não & 6 & 27,3 \\
\hline \multicolumn{3}{|c|}{ Apoio recebido do parceiro } \\
\hline Excelente/Muito bom & 7 & 31,8 \\
\hline Bom & 6 & 27,3 \\
\hline Ruim & 1 & 4,5 \\
\hline Não recebe & 8 & 36,4 \\
\hline Total & 22 & 100,0 \\
\hline
\end{tabular}

adicionais ao exercer a função de cuidador principal, além de não contarem com ajuda do parceiro $(36,4 \%)$ para prestar a assistência.

O tempo na função de cuidador variou de 15 dias a 14 anos e três meses, com média de seis anos ( $\mathrm{DP}=5,11$ meses), enquanto que as horas semanais dedicadas ao cuidado variaram de 1 a 30 horas, prevalecendo o intervalo de até 5 horas. Na Figura 2, fica notório que 27\% necessitavam de cuidados integrais.

\section{DISCUSSÃO}

Torna-se necessário conhecer o perfil sociodemográfico e clínico de cuidadores de crianças/adolescentes estomizados, pois na medida em que os resultados aparecem, criam-se ferramentas para o planejamento e a programação de ações que contemplem as necessidades da assistência aos que prestam esses cuidados.

$\mathrm{O}$ ato de cuidar é um processo que acarreta desgastes significativos à saúde do cuidador, os quais, se não considerados e amenizados, podem levá-lo à condição de cronicidade, passando de cuidador à pessoa a ser cuidada ${ }^{14}$. Neste estudo,

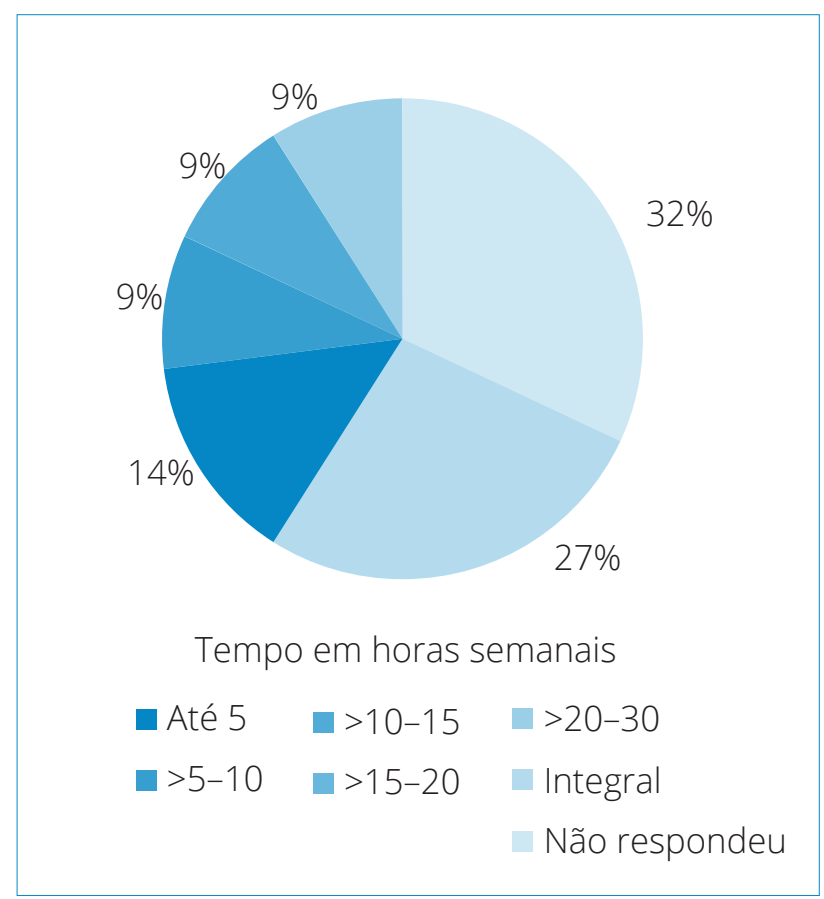

Figura 2. Distribuição de cuidadores de crianças e adolescentes estomizados atendidos em um serviço de reabilitação do Distrito Federal, de acordo com o tempo (em horas) por semana dedicadas ao cuidado. Brasília (DF), Brasil; 2013. 
verificou-se que as mães, principalmente, assumiram a função de cuidador principal, seguidas pelo pai e as avós. O cuidador representa apoio e suporte durante o tratamento e recuperação da criança/adolescente estomizado. Sua presença implica na manutenção da afetividade, oferecimento de apoio emocional e cuidado direto ao estoma. Esse apoio se reflete nos seguintes aspectos: higiene da estomia e da pele periestomia, troca, esvaziamento e higienização do equipamento coletor, observação e tratamento dos sinais de anormalidade da estomia (dermatite periestomia, eritema, erosão, pústula, úlcera, infecção, varizes periestomias) e, também, o descarte dos equipamentos coletores.

Tem-se como cuidador principal aquele que assume a responsabilidade na prestação de cuidados à saúde exigidos pelo tratamento da condição crônica e, também, o que proporciona a maior parte dos cuidados ${ }^{14}$. Essas características são similares àquelas reportadas por estudos recentes envolvendo cuidadores de pessoas com doenças crônicas, deficiências intelectuais e idosos ${ }^{14-17}$. A mulher, apesar de ter ampliado a sua participação no contexto social, ainda mantém a responsabilidade pelas atividades domésticas, tanto sobre o cuidado de si quanto o dos outros ${ }^{18}$.

Estudos de famílias de pacientes dependentes mostram que a escolha do cuidador não costuma ser ao acaso e que a opção pelos cuidados nem sempre é do cuidador, mas, muitas vezes, expressão de um desejo do paciente ou falta de outra opção. Pode ocorrer, também, de modo inesperado para um familiar que, ao se sentir responsável, assume esse cuidado, mesmo não se reconhecendo como um cuidador ${ }^{14,16-19}$.

Os cuidadores apresentaram predominância de baixa escolaridade, pois a maioria cursou o ensino fundamental incompleto. Identificar o grau de escolaridade permite adequar de maneira clara as informações sobre educação em saúde a serem fornecidas aos indivíduos. Outro fator relevante observado foi que houve predomínio de cuidadores que preferiram não trabalhar fora para cuidar do filho e os que trabalhavam relatam que já tiveram que faltar ao emprego muitas vezes por compromissos assistenciais de sua criança ou adolescente.

A maioria das famílias recebia de 1 a 3 salários mínimos, já incluindo o benefício aos deficientes cedidos pelo governo brasileiro pela condição de estomizado e o da bolsa família para os pais e/ou responsáveis que possuíam filhos que frequentassem a escola. Tal informação corrobora com o estudo desenvolvido em 2009 que avaliou a sobrecarga dos cuidadores de crianças com paralisia cerebral por meio da escala Burden Interview e mostrou correlação negativa e significativa entre o baixo nível educacional, social e econômico e a sobrecarga vivenciada pelos cuidadores ${ }^{20}$, assim como reforça a estreita relação entre anos de estudo e renda familiar, na qual a precária qualificação profissional se reflete na limitação de recursos financeiros da família.

Todos os participantes estão cadastrados no programa de assistência de enfermagem ao estomizado do DF e, por isso, recebem dispositivo coletor de modo a suprir suas necessidades mensais, pois são materiais de uso descartável. Ocorre, ainda, a aquisição de materiais adjuvantes como placas, pós, pomadas protetoras da pele e de segurança, além de orientações acerca da utilização adequada dos dispositivos, dos cuidados com o estoma, da pele e de como evitar possíveis complicações.

Os estomizados cadastrados recebem cerca de 10 a 15 unidades de dispositivos coletores com suas respectivas placas, caso sejam de duas peças, considerando o manejo recomendado dos dispositivos como mensuração do estoma, colocação e esvaziamento das bolsas, estima-se que esse quantitativo de insumos seja suficiente para um mês. Os sujeitos também referiram que mesmo com a ajuda do governo, em algum momento precisam realizar custos adicionais por necessidade de fraldas, medicamentos, alimentos ou mesmo dispositivo coletor.

Foi interessante observar que mesmo jovens, esses cuidadores referiram estar acometidos por um expressivo percentual de doenças. Mesmo aqueles que não buscaram atendimento médico no último ano, não descartaram a possibilidade de estar com algum problema de saúde. Dos que se declararam doentes, apenas alguns utilizavam medicamentos para controle de seu problema de saúde. $\mathrm{O}$ aparecimento das doenças muitas vezes pode estar relacionado à sobrecarga e/ou dedicação aos cuidados, não existindo tempo para o cuidador suprir suas necessidades pessoais, negligenciando a procura de assistência à saúde e agravando situações que poderiam ser debeladas ou amenizadas no início. Em estudo realizado em 2010, no qual foram avaliados 66 cuidadores informais de deficientes intelectuais, evidenciou-se que existe uma correlação das variáveis do cuidado e da sobrecarga na qualidade de vida dos cuidadores, sendo que quanto maior a sobrecarga apresentada, pior a qualidade de vida ${ }^{21}$. Assim, compreende-se que o grau de incapacidade e dependência do indivíduo determinam o tipo de assistência necessária, tornando-se, desse modo, um desafio ao cuidador principal. $\mathrm{Na}$ literatura acerca do assunto, termos equivalentes como 
sobrecarga, impacto e interferência são comumente utilizados para descrever as consequências do ato de cuidar ${ }^{14}$.

O tempo dedicado às atividades relacionadas ao cuidar do estomizado variou bastante, e constou da rotineira troca do equipamento coletor à higiene corporal completa. Em especial, com as crianças e adolescentes estomizados esse tempo pode estar relacionado à aquisição da autonomia para assumir seu próprio cuidado. Houve relato de cuidador que conferia ajuda somente quando solicitado, pois, na maioria das vezes, o estomizado já havia realizado a atividade sozinho. Nesse caso, os pais e/ou cuidadores exercem a função de mediadores na construção do autocuidado, importante papel para a autonomia dessas pessoas.

Ressalta-se, também, que o tempo gasto para realizar o cuidado poderá oscilar à medida que o cuidador adquirir habilidades nas atividades necessárias. Os cuidadores que prestavam cuidados integrais, devido ao grau de dependência do filho, necessitavam de maior tempo de dedicação. Quando se trata de crianças em idade pré-escolar, muitas vezes, é o enfermeiro estomaterapeuta quem direciona os pais no estabelecimento de rotinas de troca de dispositivos, cuidados em relação à estomia e sobre o gerenciamento desses cuidados em casa. Durante a troca ou cuidado deve-se estimular o autocuidado gradativamente, respeitando a habilidade individual do paciente ${ }^{22}$.

Em estudo com cuidadores de pacientes crônicos, concluiu-se que a maneira como o cuidador prestava os cuidados estava ligado ao modo como ele entendia o ato de cuidar. Outro ponto estudado é colaborar com o cuidador por meio de divisão de responsabilidades, aceitação de apoio de terceiros e estratégias de revezamento que ajudam a diminuir a sobrecarga que o cuidador assume, possibilitando o seu retorno às atividades de lazer, trabalho e autocuidado ${ }^{14}$.

As limitações para o estudo foram: primeiro, a metodologia adotada, pois estudos descritivos são realizados em um período definido. Outra limitação significativa para o estudo foi ter sido realizado em apenas um centro de estudo, porém esse local é referência nesse tipo de atendimento, no Distrito Federal.

\section{CONCLUSÃO}

Este estudo evidenciou as características dos cuidadores de crianças e adolescentes cujos dados sociodemográficos principais foram: predominância feminina, idade média de 41,27 anos, casadas, pardas, com ensino fundamental, procedentes de áreas administrativas do DF e que os cuidadores podem apresentar problemas na saúde física e emocional em decorrência de priorizarem o cuidado da criança e/ou adolescente estomizado.

Em relação à caracterização clínica, a maioria tinha algum problema de saúde, embora uma pequena parcela tenha procurado atendimento médico nos últimos anos, e faziam uso de medicamentos. A mãe é o cuidador principal, recebe ajuda do governo, possui custos adicionais na função de cuidador e gasta em média de 5 a 30 horas semanais prestando cuidados.

Até a criança e/ou adolescente adquirir autonomia para o autocuidado, o cuidador é quem realiza todas as atividades inerentes ao cuidado com a estomia: essa condição explica sua importância e definição de cuidador principal. Vale mencionar que toda ação de cuidado visa promover a melhor qualidade de vida ao paciente, porém o cuidador precisar ser considerado como elo essencial para a promoção, assistência e reabilitação desses pacientes.

Evidencia-se, portanto, que há necessidade de ampliar os estudos na área, pois o cuidador precisa ser valorizado e reconhecido como um elemento importante da prestação de cuidado à saúde.

A obtenção das informações colhidas nesta pesquisa poderá subsidiar profissionais de saúde na assistência aos cuidadores e estomizados, além de oferecer qualidade no cuidado à criança e ao adolescente com estomia.

\section{REFERÊNCIAS}

1. Souza JL, Gomes GC, Barros EJL. O cuidado à pessoa portadora de estomia: o papel do familiar cuidador. Rev. enferm. UERJ. 2009;17(4):550-5.

2. Pacilli M, Eaton S, Fell JM, Rawat D, Clarke S, Haddad MJ. Surgery in children with Crohn disease refractory to medical therapy. J Pediatr Gastroenterol Nutr. 2011;52(3):286-90.
3. Monteiro SNC, Kamada I, SilvaA L, Souza TCR. Perfil de Crianças e Adolescentes Estomizados Atendidos de um Hospital Público do Distrito Federal. Rev Estima. 2014;12 (3):23 - 32.

4. Saklani AP, Marsden N, Davies M, Carr ND, Beynon J. Outcome after restorative proctocolectomy in children and adolescents. Colorectal Dis. 2011;13(10):1148-52. 
5. Coldicutt P, Hill B. An overview of surgical stoma construction and its effects on the child and their family. Nurs child young people. 2013;25(4):26-35.

6. Pena A. Migotto-kriegerb M, Levitt MA. Colostomy in anorectal malformations: a procedure with serious but preventable complications. J pediatr surg. 2008;41(4):748-56.

7. Brasília. Secretaria de Estado de Saúde do Distrito Federal (SES/DF). Cadastro de Pacientes no Núcleo de Atenção ao Estomizado. Brasília: Estatística; 2012.

8. Guerrero S, Angelo M. Impacto del estoma enteral en el niño y la familia. av.enferm. 2010; XXVIII (especial):99-108.

9. Oliveira FS, Vasconcelos VM, Martins MC, Lúcio IML. Cuidado à criança com distrofia muscular dependente de tecnologia no domicílio: concepção de mães. Rev Rene. 2013;14(1):82-91.

10. Melo MC, Kamada I. Anomalia anorretal e cuidados maternos. Rev Bras Enferm. 2011; 64(1):176-9.

11. Susin FP, Bortolini V, Sukiennik R, Mancopes R, Barbosa LR. Perfil de pacientes com paralisia cerebral em uso de gastrostomia e efeito nos cuidadores. Rev CEFAC. 2012;14(5):933-42.

12. Barbosa MAM, Balieiro MMFG, Pettengill MAM. Cuidado centrado na família no contexto da criança com deficiência e sua família: uma análise reflexiva. Texto Contexto. 2012;21(1):194-99.

13. Statístical Package for the Social Sciences [internet]. [22 jan 2015]. SPSS step-bystep. Tutorial: part 1. DataStep Development; 2004. Available from: www.datastep.com
14. Brito DCS. Cuidando de quem cuida: estudo de caso sobre o cuidador principal de um portador de insuficiência renal crônica. Psicol Estud. 2009;14(3):603-07.

15. Veiga Junior NN, Ciasca SM, Rodrigues SD. Qualidade de vida de cuidadores de crianças com PC ou AVC: Estudo comparativo. Rev Neurocienc. 2011;19(4):602-08.

16. Nobrega IM, Pereira CU. Qualidade de vida, ansiedade e depressão em cuidadores de crianças com neoplasia cerebral. Psicol Teor Prat. 2011;13(1):48-61.

17. Fernandes CPL, Vale DEASC, Nóbrega EBS, Dias MMS, Sousa SEF. Ansiedade e necessidades dos cuidadores de crianças com e sem deficiência. Rev Enf Ref. 2012;III(6): 181-89.

18. Santos LF, Oliveira LMAC, Barbosa MA, Siqueira KM, Peixoto MKAV. Reflexos da hospitalização da criança na vida do familiar acompanhante. Rev Bras Enferm. 2013;66(4):473-78.

19. Ribeiro AF, Souza CAD. O cuidador familiar de doentes com câncer. Arq ciênc saúde. 2010;17(1):22-27.

20. Camargos ACR, de Lacerda TTB, Viana SO, Pinto LRA, Fonseca MLS. Avaliação da sobrecarga do cuidador de crianças com paralisia cerebral através da escala Burden Interview. Rev Bras Saúde Matern Infant. 2009;9(1):31-7.

21. Pimenta RA, Rodrigues LA, Greguol M. Avaliação da Qualidade de vida e sobrecarga de cuidadores de pessoas com deficiência Intelectual. Rev bras cienc saúde. 2010;14(3):69-76.

22. Poletto D, Gonçalves MI, Barros MTT, Anders JC, Martins ML. A criança com estoma intestinal e sua família: implicações para o cuidado de enfermagem. Texto contexto. 2011;20(2):319-27. 